\title{
New extensions concerned with results by Ponnusamy and Karunakaran
}

\author{
Mamoru Nunokawa ${ }^{1}$, Kazuo Kuroki ${ }^{2}$, Janusz Sokół ${ }^{3}$ and Shigeyoshi Owa ${ }^{2 *}$
}

${ }^{\text {*} C o r r e s p o n d e n c e: ~}$
shige21@ican.zaq.ne.jp
${ }^{2}$ Department of Mathematics, Kinki
University, Higashi-Osaka, Osaka
$577-8502$, Japan
Full list of author information is
available at the end of the article

available at the end of the article

\author{
Abstract \\ A subclass $\mathcal{A}(n, k)$ of analytic functions $f(z)$ in the open unit disk $\mathbb{U}$ is introduced. By \\ means of the result due to Fukui and Sakaguchi (Bull. Fac. Edu. Wakayama Univ. Natur. \\ Sci. 30:1-3, 1980), some interesting properties of $f(z)$ in $\mathcal{A}(n, k)$ concerned with \\ Ponnusamy and Karunakaran (Complex Var. Theory Appl. 11:79-86, 1989) are \\ discussed. \\ MSC: Primary 30C45 \\ Keywords: analytic; starlike; Jack's lemma
}

\section{Introduction}

Let $\mathcal{A}(n, k)$ be a class of functions $f(z)$ of the form

$$
f(z)=z^{n}+\sum_{m=n+k}^{\infty} a_{m} z^{m} \quad(n \geqq 1, k \geqq 1)
$$

which are analytic in the open unit disk $\mathbb{U}=\{z \in \mathbb{C}:|z|<1\}$. For two functions $f(z)$ and $g(z)$ belonging to the class $\mathcal{A}(1,1)$, Sakaguchi [1] proved the following result.

Theorem A Let $f(z) \in \mathcal{A}(1,1)$ and $g(z) \in \mathcal{A}(1,1)$ be starlike in $\mathbb{U}$. If $f(z)$ and $g(z)$ satisfy

$$
\operatorname{Re}\left(\frac{f^{\prime}(z)}{g^{\prime}(z)}\right)>0 \quad(z \in \mathbb{U})
$$

then

$$
\operatorname{Re}\left(\frac{f(z)}{g(z)}\right)>0 \quad(z \in \mathbb{U})
$$

After Theorem A, many mathematicians studying this field have applied this theorem to get some results (see [2]). In 1989, Ponnusamy and Karunakaran [3] improved Theorem A as follows.

Theorem B Let $\alpha$ be a complex number with $\operatorname{Re} \alpha>0$ and $\beta<1$. Further, let $f(z) \in \mathcal{A}(n, k)$ and $g(z) \in \mathcal{A}(n, j)(j \geqq 1)$ satisfy

$$
\operatorname{Re}\left(\frac{\alpha g(z)}{z g^{\prime}(z)}\right)>\delta \quad(z \in \mathbb{U})
$$


with $0 \leqq \delta<\frac{\operatorname{Re} \alpha}{n}$. Iff $(z)$ and $g(z)$ satisfy

$$
\operatorname{Re}\left\{(1-\alpha) \frac{f(z)}{g(z)}+\alpha \frac{f^{\prime}(z)}{g^{\prime}(z)}\right\}>\beta \quad(z \in \mathbb{U})
$$

then

$$
\operatorname{Re}\left(\frac{f(z)}{g(z)}\right)>\frac{2 \beta+\delta k}{2+\delta k} \quad(z \in \mathbb{U}) .
$$

It is the purpose of the present paper to discuss Theorem B applying the lemma due to Fukui and Sakaguchi [4]. To discuss our problems, we need the following lemmas.

Lemma 1 Let $w(z)=\sum_{n=k}^{\infty} a_{n} z^{n}\left(a_{k} \neq 0, k \geqq 1\right)$ be analytic in $\mathbb{U}$. If the maximum value of $|w(z)|$ on the circle $|z|=r<1$ is attained at $z=z_{0}$, then we have

$$
\frac{z_{0} w^{\prime}\left(z_{0}\right)}{w\left(z_{0}\right)}=\ell \geqq k,
$$

which shows that $\frac{z_{0} w^{\prime}\left(z_{0}\right)}{w\left(z_{0}\right)}$ is a positive real number.

The proof of Lemma 1 can be found in [4], and we see that Lemma 1 is a generalization of Jack's lemma given by Jack [5]. Applying Lemma 1, we derive the following.

Lemma 2 Let $p(z)=1+\sum_{n=k}^{\infty} c_{n} z^{n}\left(c_{k} \neq 0, k \geqq 1\right)$ be analytic in $\mathbb{U}$ with $p(z) \neq 0(z \in \mathbb{U})$. If there exists a point $z_{0} \in \mathbb{U}$ such that

$$
\operatorname{Re} p(z)>0 \quad\left(|z|<\left|z_{0}\right|\right)
$$

and

$$
\operatorname{Re} p\left(z_{0}\right)=0,
$$

then we have

$$
-z_{0} p^{\prime}\left(z_{0}\right) \geqq \frac{\ell}{2}\left(1+\left|p\left(z_{0}\right)\right|^{2}\right),
$$

and so

$$
\frac{z_{0} p^{\prime}\left(z_{0}\right)}{p\left(z_{0}\right)}=i \ell
$$

where

$$
k \leqq \frac{k}{2}\left(a+\frac{1}{a}\right) \leqq \ell \quad\left(\arg p\left(z_{0}\right)=\frac{\pi}{2}\right)
$$

and

$$
-k \geqq-\frac{k}{2}\left(a+\frac{1}{a}\right) \geqq \ell \quad\left(\arg p\left(z_{0}\right)=-\frac{\pi}{2}\right)
$$

with $p\left(z_{0}\right)= \pm i a(a>0)$. 
Proof Let us consider

$$
\phi(z)=\frac{1-p(z)}{1+p(z)}=\frac{c_{k}}{2} z^{k}+\cdots
$$

for $p(z)$. Then, it follows that $\phi(0)=\phi^{\prime}(0)=\cdots=\phi^{(k-1)}(0)=0,|\phi(z)|<1\left(|z|<\left|z_{0}\right|\right)$ and $\left|\phi\left(z_{0}\right)\right|=1$. Therefore, applying Lemma 1 , we have that

$$
\frac{z_{0} \phi^{\prime}\left(z_{0}\right)}{\phi\left(z_{0}\right)}=\frac{-2 z_{0} p^{\prime}\left(z_{0}\right)}{1-\left(p\left(z_{0}\right)\right)^{2}}=\frac{-2 z_{0} p^{\prime}\left(z_{0}\right)}{1+\left|p\left(z_{0}\right)\right|^{2}}=\ell \geqq k .
$$

This implies that $z_{0} p^{\prime}\left(z_{0}\right)$ is a negative real number and

$$
-z_{0} p^{\prime}\left(z_{0}\right) \geqq \frac{k}{2}\left(1+\left|p\left(z_{0}\right)\right|^{2}\right)
$$

Let us use the same method by Nunokawa [6]. If $\arg p\left(z_{0}\right)=\frac{\pi}{2}$, then we write $p\left(z_{0}\right)=i a$ $(a>0)$. This gives us that

$$
\operatorname{Im}\left(\frac{z_{0} p^{\prime}\left(z_{0}\right)}{p\left(z_{0}\right)}\right)=\operatorname{Im}\left(-\frac{i z_{0} p^{\prime}\left(z_{0}\right)}{a}\right) \geqq \frac{k}{2}\left(a+\frac{1}{a}\right) .
$$

If $\arg p\left(z_{0}\right)=-\frac{\pi}{2}$, then we write $p\left(z_{0}\right)=-i a(a>0)$. Thus we have that

$$
\operatorname{Im}\left(\frac{z_{0} p^{\prime}\left(z_{0}\right)}{p\left(z_{0}\right)}\right)=\operatorname{Im}\left(\frac{i z_{0} p^{\prime}\left(z_{0}\right)}{a}\right) \leqq-\frac{k}{2}\left(a+\frac{1}{a}\right) .
$$

This completes the proof of Lemma 2.

\section{Main results}

With the help of Lemma 2, we derive the following theorem.

Theorem 1 Let $\alpha$ be a complex number with $\operatorname{Re} \alpha>0$ and $\beta<1$. Further, let $f(z) \in \mathcal{A}(n, k)$ and $g(z) \in \mathcal{A}(n, j)(j \geqq 1)$ satisfy

$$
\operatorname{Re}\left(\frac{\alpha g(z)}{z g^{\prime}(z)}\right)>\delta \quad(z \in \mathbb{U})
$$

with $0 \leqq \delta<\frac{\operatorname{Re} \alpha}{n}$. Iff $(z)$ and $g(z)$ satisfy

$$
\operatorname{Re}\left\{(1-\alpha) \frac{f(z)}{g(z)}+\alpha \frac{f^{\prime}(z)}{g^{\prime}(z)}\right\}+\frac{\delta k}{2\left(1-\beta_{1}\right)}\left|\frac{f(z)}{g(z)}-\beta_{1}\right|^{2}>\beta \quad(z \in \mathbb{U}),
$$

then

$$
\operatorname{Re}\left(\frac{f(z)}{g(z)}\right)>\beta_{1} \quad(z \in \mathbb{U})
$$

where $\beta_{1}=\frac{2 \beta+\delta k}{2+\delta k}$. 
Proof Defining the function $p(z)$ by

$$
p(z)=\frac{\frac{f(z)}{g(z)}-\beta_{1}}{1-\beta_{1}},
$$

we see that $p(0)=1$ and

$$
\begin{aligned}
& \operatorname{Re}\left\{(1-\alpha) \frac{f(z)}{g(z)}+\alpha \frac{f^{\prime}(z)}{g^{\prime}(z)}-\beta\right\} \\
& =\operatorname{Re}\left\{\left(\beta_{1}-\beta\right)+\left(1-\beta_{1}\right)\left(p(z)+\frac{\alpha g(z)}{z g^{\prime}(z)} z p^{\prime}(z)\right)\right\} \\
& >-\frac{\delta k}{2\left(1-\beta_{1}\right)}\left|\frac{f(z)}{g(z)}-\beta_{1}\right|^{2}
\end{aligned}
$$

for all $z \in \mathbb{U}$. Let us suppose that there exists a point $z_{0} \in \mathbb{U}$ such that

$$
\left|\arg p\left(z_{0}\right)\right|<\frac{\pi}{2} \quad\left(|z|<\left|z_{0}\right|\right)
$$

and

$$
\left|\arg p\left(z_{0}\right)\right|=\frac{\pi}{2}
$$

Then, by means of Lemma 2 , we have that

$$
-z_{0} p^{\prime}\left(z_{0}\right) \geqq \frac{k}{2}\left(1+\left|p\left(z_{0}\right)\right|^{2}\right)
$$

If follows from the above that

$$
\begin{aligned}
& \operatorname{Re}\left\{(1-\alpha) \frac{f\left(z_{0}\right)}{g\left(z_{0}\right)}+\alpha \frac{f^{\prime}\left(z_{0}\right)}{g^{\prime}\left(z_{0}\right)}-\beta\right\} \\
& =\left(\beta_{1}-\beta\right)+\left(1-\beta_{1}\right) \operatorname{Re}\left\{p\left(z_{0}\right)+\frac{\alpha g\left(z_{0}\right)}{z_{0} g^{\prime}\left(z_{0}\right)} z_{0} p^{\prime}\left(z_{0}\right)\right\} \\
& =\left(\beta_{1}-\beta\right)-\left(1-\beta_{1}\right) \operatorname{Re}\left\{\frac{\alpha g\left(z_{0}\right)}{z_{0} g^{\prime}\left(z_{0}\right)}\left(-z_{0} p^{\prime}\left(z_{0}\right)\right)\right\} \\
& \leqq\left(\beta_{1}-\beta\right)-\left(1-\beta_{1}\right) \frac{\delta k}{2}\left(1+\left|p\left(z_{0}\right)\right|^{2}\right) \\
& =-\frac{\delta k}{2\left(1-\beta_{1}\right)}\left|\frac{f\left(z_{0}\right)}{g\left(z_{0}\right)}-\beta_{1}\right|^{2},
\end{aligned}
$$

which contradicts (2.5). This completes the proof of the theorem.

Remark 1 If $f(z)$ and $g(z)$ satisfy $f(z)=\beta_{1} g(z)$ in Theorem 1 , then Theorem 1 becomes Theorem B given by Ponnusamy and Karunakaran [3]. We also have the following theorem.

Theorem 2 Let $\alpha$ be a complex number with $\operatorname{Re} \alpha>0$ and $\beta<1$. Further, let $f(z) \in \mathcal{A}(n, k)$ and $g(z) \in \mathcal{A}(n, j)(j \geqq 1)$ satisfy the condition (2.1) with $0 \leqq \delta<\frac{\operatorname{Re} \alpha}{n} \leqq 1+\delta$. Iff $(z)$ and $g(z)$ 
satisfy

$$
\left|\arg \left\{(1-\alpha) \frac{f(z)}{g(z)}+\alpha \frac{f^{\prime}(z)}{g^{\prime}(z)}-\beta\right\}\right|<\frac{\pi}{2}+\operatorname{Tan}^{-1}\left(\frac{\delta k|p(z)|}{2\left(\frac{2 r}{1-r^{2}}+\frac{|\operatorname{Im} \alpha|}{n}+1\right)}\right)
$$

for $|z|=r<1$, then

$$
\left|\arg \left(\frac{f(z)}{g(z)}-\beta_{1}\right)\right|<\frac{\pi}{2} \quad(z \in \mathbb{U})
$$

or

$$
\operatorname{Re}\left(\frac{f(z)}{g(z)}\right)>\beta_{1} \quad(z \in \mathbb{U})
$$

where $\beta_{1}=\frac{2 \beta+\delta k}{2+\delta k}$ and

$$
p(z)=\frac{\frac{f(z)}{g(z)}-\beta_{1}}{1-\beta_{1}} .
$$

Proof Note that the function $p(z)$ is analytic in $\mathbb{U}$ and $p(0)=1$. It follows that

$$
\begin{aligned}
\left|\arg \left\{(1-\alpha) \frac{f(z)}{g(z)}+\alpha \frac{f^{\prime}(z)}{g^{\prime}(z)}-\beta\right\}\right| & =\left|\arg \left\{\left(\beta_{1}-\beta\right)+\left(1-\beta_{1}\right)\left(p(z)+\frac{\alpha g(z)}{z g^{\prime}(z)} z p^{\prime}(z)\right)\right\}\right| \\
& <\frac{\pi}{2}+\operatorname{Tan}^{-1}\left(\frac{\delta k|p(z)|}{2\left(\frac{2 r}{1-r^{2}}+\frac{|\operatorname{Im} \alpha|}{n}+1\right)}\right)
\end{aligned}
$$

for $|z|=r<1$. If there exists a point $z_{0} \in \mathbb{U}$ such that

$$
\left|\arg p\left(z_{0}\right)\right|<\frac{\pi}{2} \quad\left(|z|<\left|z_{0}\right|\right)
$$

and

$$
\left|\arg p\left(z_{0}\right)\right|=\frac{\pi}{2}
$$

then, by Lemma 2, we have that

$$
\frac{z_{0} p^{\prime}\left(z_{0}\right)}{p\left(z_{0}\right)}=i \ell
$$

where

$$
\frac{k}{2}\left(a+\frac{1}{a}\right) \leqq \ell \quad\left(\arg p\left(z_{0}\right)=\frac{\pi}{2}\right)
$$

and

$$
-\frac{k}{2}\left(a+\frac{1}{a}\right) \geqq \ell \quad\left(\arg p\left(z_{0}\right)=-\frac{\pi}{2}\right)
$$


with $p\left(z_{0}\right)= \pm i a(a>0)$. If $\arg p\left(z_{0}\right)=\frac{\pi}{2}$, then it follows that

$$
\begin{aligned}
& \arg \left\{(1-\alpha) \frac{f\left(z_{0}\right)}{g\left(z_{0}\right)}+\alpha \frac{f^{\prime}\left(z_{0}\right)}{g^{\prime}\left(z_{0}\right)}-\beta\right\} \\
& =\arg p\left(z_{0}\right)\left\{\frac{\beta_{1}-\beta}{p\left(z_{0}\right)}+\left(1-\beta_{1}\right)\left(1+\frac{\alpha g\left(z_{0}\right)}{z_{0} g^{\prime}\left(z_{0}\right)} \frac{z_{0} p^{\prime}\left(z_{0}\right)}{p\left(z_{0}\right)}\right)\right\} \\
& =\frac{\pi}{2}+\arg \left\{-\left(\frac{\beta_{1}-\beta}{a}\right) i+\left(1-\beta_{1}\right)\left(1+i \ell \frac{\alpha g\left(z_{0}\right)}{z_{0} g^{\prime}\left(z_{0}\right)}\right)\right\} \\
& =\frac{\pi}{2}+\arg I\left(z_{0}\right),
\end{aligned}
$$

where

$$
I\left(z_{0}\right)=-\left(\frac{\beta_{1}-\beta}{a}\right) i+\left(1-\beta_{1}\right)\left(1+i \ell \frac{\alpha g\left(z_{0}\right)}{z_{0} g^{\prime}\left(z_{0}\right)}\right) .
$$

Note that

$$
\begin{aligned}
\operatorname{Im} I\left(z_{0}\right) & =\frac{\beta-\beta_{1}}{a}+\left(1-\beta_{1}\right) \ell \operatorname{Re} \frac{\alpha g\left(z_{0}\right)}{z_{0} g^{\prime}\left(z_{0}\right)} \\
& \geqq\left(1-\beta_{1}\right) \delta \ell+\frac{\beta-\beta_{1}}{a} \\
& \geqq \frac{\delta k}{2}\left(1-\beta_{1}\right)\left(a+\frac{1}{a}\right)+\frac{\beta-\beta_{1}}{a} \\
& =\frac{\delta k}{2}\left(1-\beta_{1}\right) a>0
\end{aligned}
$$

and

$$
\operatorname{Re} I\left(z_{0}\right)=\left(1-\beta_{1}\right)\left(1-\ell \operatorname{Im}\left(\frac{\alpha g\left(z_{0}\right)}{z_{0} g^{\prime}\left(z_{0}\right)}\right)\right) \leqq\left(1-\beta_{1}\right)\left(1+\ell\left|\operatorname{Im}\left(\frac{\alpha g\left(z_{0}\right)}{z_{0} g^{\prime}\left(z_{0}\right)}\right)\right|\right)
$$

Letting

$$
q(z)=\frac{\alpha g(z)}{z g^{\prime}(z)}+1-\frac{\alpha}{n}
$$

we know that $q(z)$ is analytic in $\mathbb{U}$ with $q(0)=1$ and $\operatorname{Re} q(z)>0(z \in \mathbb{U})$. Therefore, applying the subordinations, we can write that

$$
q(z)=\frac{1-w(z)}{1+w(z)}
$$

with the Schwarz function $w(z)$ analytic in $\mathbb{U}, w(0)=0$ and $|w(z)| \leqq|z|$. This leads us to

$$
|w(z)|=\left|\frac{1-q(z)}{1+q(z)}\right| \leqq r \quad(|z| \leqq r<1)
$$

which is equivalent to

$$
\left|q(z)-\frac{1+r^{2}}{1-r^{2}}\right| \leqq \frac{2 r}{1-r^{2}}
$$


This gives us that

$$
|\operatorname{Im} q(z)|=\left|\operatorname{Im}\left(\frac{\alpha g(z)}{z g^{\prime}(z)}+1-\frac{\alpha}{n}\right)\right| \leqq \frac{2 r}{1-r^{2}}
$$

for $|z|=r<1$. Thus we have that

$$
\left|\operatorname{Im}\left(\frac{\alpha g\left(z_{0}\right)}{z_{0} g^{\prime}\left(z_{0}\right)}\right)\right| \leqq \frac{2 r}{1-r^{2}}+\frac{|\operatorname{Im} \alpha|}{n} \quad(|z|=r<1) .
$$

Using (2.12) and (2.15), we obtain that

$$
\arg I\left(z_{0}\right)=\operatorname{Tan}^{-1}\left(\frac{\operatorname{Im} I\left(z_{0}\right)}{\operatorname{Re} I\left(z_{0}\right)}\right) \geqq \operatorname{Tan}^{-1}\left(\frac{\delta k a}{2\left(\frac{2 r}{1-r^{2}}+\frac{|\operatorname{Im} \alpha|}{n}+1\right)}\right)
$$

which contradicts our condition (2.7).

If $\arg p\left(z_{0}\right)=-\frac{\pi}{2}$, using the same way, we also have that

$$
\arg \left\{(1-\alpha) \frac{f\left(z_{0}\right)}{g\left(z_{0}\right)}+\alpha \frac{f^{\prime}\left(z_{0}\right)}{g^{\prime}\left(z_{0}\right)}-\beta\right\} \leqq-\left\{\frac{\pi}{2}+\operatorname{Tan}^{-1}\left(\frac{\delta k a}{2\left(\frac{2 r}{1-r^{2}}+\frac{|\operatorname{Im} \alpha|}{n}+1\right)}\right)\right\}
$$

which contradicts (2.7)

\section{Competing interests}

The authors did not provide this information.

\section{Authors' contributions}

The authors did not provide this information.

\section{Author details}

${ }^{1}$ University of Gunma, 798-8 Hoshikuki, Chuou-Ward, Chiba 260-0808, Japan. ${ }^{2}$ Department of Mathematics, Kinki University, Higashi-Osaka, Osaka 577-8502, Japan. ${ }^{3}$ Department of Mathematics, Rzeszow University of Technology, Al. Powstańców, Warszawy 12, Rzeszów, 35-959, Poland.

\section{Acknowledgements}

Dedicated to Professor Hari M Srivastava.

Received: 11 January 2013 Accepted: 22 April 2013 Published: 9 May 2013

\section{References}

1. Sakaguchi, K: On a certain univalent mapping. J. Math. Soc. Jpn. 11, $72-75$ (1959)

2. Srivastava, HM, Owa, S (eds.): Current Topics in Analytic Function Theory. World Scientific, Singapore (1992)

3. Ponnusamy, S, Karunakaran, V: Differential subordination and conformal mappings. Complex Var. Theory Appl. 11, 79-86 (1989)

4. Fukui, S, Sakaguchi, K: An extension of a theorem of S. Ruscheweyh. Bull. Fac. Ed. Wakayama Univ. Natur. Sci. 30, 1-3 (1980)

5. Jack, IS: Functions starlike and convex of order $\alpha$. J. Lond. Math. Soc. 2, 469-474 (1971)

6. Nunokawa, M: On properties of non-Carathéodory functions. Proc. Jpn. Acad. 68, 152-153 (1992) 